\section{Epidemiologia das pregas cutâneas triciptal e subescapular elevadas em adolescentes}

\author{
Epidemiology of elevated triciptal and subscapular \\ skinfolds in adolescents
}

\footnotetext{
1 Programa de Pós-graduação em Epidemiologia, Universidade Federal de Pelotas, Pelotas, Brasil. 2 Universidade Estadual de Londrina, Londrina, Brasil.

Correspondência R. P. Duquia

Programa de Pós-graduação em Epidemiologia, Universidade Federal de Pelotas.

Av. Duque de Caxias 250, 3o piso, Pelotas, $R S$ 96030-002, Brasil. rodrigoduquia@terra.com.br
}

\begin{abstract}
The objective of this study was to describe and compare the tricipital (TSF) and subscapular skinfold (SSF) thickness according to demographic, socioeconomic, behavioral, and biological characteristics in adolescents from Pelotas, Rio Grande do Sul State, Brazil. A cross-sectional study nested in a birth cohort ( $n=4,452$; mean age $=11$ years) was conducted. The outcomes were defined as skinfold thickness $\geq 90^{\text {th }}$ percentile of the National Center for Health Statistics reference curve. The prevalence rates for elevated TSF and SSF were $20.2 \%$ and $17.3 \%$, respectively, in boys and $14.2 \%$ and $10.5 \%$ in girls. The strongest factor associated with adiposity in boys was socioeconomic status $(p<0.001)$, whereas among girls it was maternal body mass index $(p<0.001)$. Low physical activity (<300 minutes/week) was associated with elevated SSF only among girls, while schooling was associated with increased TSF and SSF only among boys. Diet, skin color, and sedentary behavior were not associated with any of the outcomes. We concluded that the main predictors of adiposity were maternal and socioeconomic characteristics. We recommend that further studies on this issue apply other methods to estimate body composition in order to confirm our results.
\end{abstract}

Skinfold Thickness; Anthropometry; Obesity; Adolescent

\author{
Rodrigo Pereira Duquia ${ }^{1}$ \\ Samuel de Carvalho Dumith 1 \\ Felipe Fossati Reichert 1,2 \\ Samanta Winck Madruga 1 \\ Luciano Nunes Duro 1 \\ Ana Maria Baptista Menezes 1 \\ Cora Luiza Araújo 1
}

\section{Introdução}

O sobrepeso e a obesidade consistem, atualmente, em um importante problema de saúde pública. O acúmulo excessivo de gordura está associado ao aumento no risco de desenvolvimento de diversas morbidades 1 , gerando altos custos sociais e econômicos. Indivíduos acima do peso, especialmente quando apresentam excesso de gordura na região do tronco, estão mais propensos a apresentarem um conjunto de anormalidades, agrupadas sob o rótulo de síndrome metabólica 2 .

No Brasil, a prevalência de excesso de peso entre adolescentes do sexo feminino aumentou de 7,5\% em 1974-1975 para 13,8\% em 1989 e $15,4 \%$ em 2002-2003 3. Entre os adolescentes do sexo masculino o aumento foi de 3,9\% para $8,3 \%$ e finalmente para $18 \%$ no mesmo período ${ }^{3}$. O excesso de gordura adquirido durante a adolescência tende a persistir na idade adulta, e está associado a diversos fatores de risco para doenças crônicas, tais como: hipertensão, dislipidemia e resistência insulínica 4 .

Diversos métodos de determinação da obesidade estão apresentados na literatura ${ }^{5}$. Estudos utilizando medidas antropométricas como, por exemplo, pregas cutâneas, são úteis para avaliar o estado nutricional e detectar distúrbios relacionados à composição corporal, tais como desnutrição e obesidade 6 . 
As pregas cutâneas, embora não sejam o padrão-ouro para avaliar adiposidade, são melhores indicadores da quantidade da gordura corporal do que o índice de massa corporal (IMC) 7,8. Além disso, as pregas cutâneas podem ser mensuradas em estudos com grandes amostras a um custo relativamente baixo. A prega cutânea subescapular (PCS) pode ser utilizada como indicador da adiposidade central, enquanto que a prega cutânea triciptal (PCT) indica acúmulo de gordura periférica 9,10 .

Embora alguns estudos demonstrem que a obesidade central é mais prejudicial à saúde do que a gordura periférica 11,12,13, é importante analisar os fatores associados a cada um dos padrões de adiposidade separadamente. Sendo assim, estudos que investiguem fatores associados à adiposidade na adolescência são necessários para a identificação de grupos em maior risco, e para o planejamento de intervenções destinadas ao combate e à prevenção desse agravo à saúde.

O objetivo deste estudo foi descrever e comparar a epidemiologia da PCT e da PCS elevadas no que se refere a características demográficas, sócio-econômicas, comportamentais e biológicas de adolescentes brasileiros. Para tal, foi realizado um estudo transversal aninhado a uma coorte de nascimentos na cidade de Pelotas, Rio Grande do Sul, Brasil.

\section{Metodologia}

Pelotas localiza-se no extremo Sul do Brasil e tem aproximadamente 320 mil habitantes, dos quais, $93,4 \%$ moram na zona urbana. A amostra do estudo foi composta por adolescentes pertencentes à coorte de nascimentos de 1993, detalhadamente descrita recentemente 14 . Durante o ano de 2004 e início de 2005, todos os indivíduos que compunham a coorte inicial $(\mathrm{N}=5.249)$ foram procurados para um novo acompanhamento.

As PCT e PCS foram coletadas utilizando-se plicômetro científico da marca Cescorf® (Cescorf Equipamentos Antropométricos, Porto Alegre, Brasil), conforme as recomendações de Lohman 15. Ambas as pregas foram dicotomizadas no valor correspondente ao percentil 90 da distribuição de referência para sexo e idade de acordo com a curva do National Center for Health Statistics (NCHS) 16. Foram considerados como desfechos para este estudo apresentar PCT ou PCS elevada conforme o critério mencionado acima 16

As variáveis independentes investigadas foram: cor da pele (observada pela entrevistadora); nível econômico (classificado de acordo com o critério da Associação Brasileira de Empresas de
Pesquisa - ABEP 17, a qual leva em conta a presença de bens de consumo, empregado/a mensalista no domicílio e a escolaridade do chefe da família); IMC atual da mãe (baseado em peso e altura auto-referidos); escolaridade do adolescente (em anos completos de estudo); baixa atividade física (prática inferior a 300 minutos por semana de atividades físicas 18; comportamento sedentário (quatro horas ou mais por dia assistindo à televisão, videogame ou computador) e dieta inadequada (baixo consumo de fibras e alto consumo de gorduras na alimentação) conforme o questionário de freqüência alimentar de Block 19. Esse questionário atribui pontos para a freqüência de ingestão dos alimentos. A soma dessa pontuação resulta em um escore de consumo de fibras e de gordura. Adolescentes que alcançaram escore inferior a 20 pontos em relação aos alimentos ricos em fibra e aqueles que somaram mais de 27 pontos em alimentos ricos em gordura foram classificados como tendo dieta inadequada.

Os dados foram coletados por entrevistadores do sexo feminino com pelo menos 18 anos de idade e nível médio de escolaridade. As entrevistadoras foram previamente treinadas para a correta mensuração das pregas cutâneas e aplicação dos questionários.

As entrevistadoras foram submetidas a testes de padronização, antes de iniciarem o trabalho de campo, para avaliação da repetibilidade e validade das medidas. Foram considerados aceitáveis erros técnicos intra-observadores inferiores a $0,80 \mathrm{~mm}$ para a PCT e inferiores a $1,83 \mathrm{~mm}$ para a PCS. Quanto aos erros técnicos interobservadores, aceitou-se como máximos 1,89mm e $1,53 \mathrm{~mm}$ para as PCT e PCS, respectivamente 20. Essa padronização baseou-se no Caderno de Atenção Básica do Ministério da Saúde 21. Ao longo da coleta de dados, que durou cerca de um ano, as entrevistadoras foram avaliadas a cada dois meses quanto às técnicas de mensuração das pregas cutâneas e de aplicação do questionário. Para fins de controle de qualidade, uma versão reduzida do questionário foi reaplicada a $10 \%$ da amostra, aleatoriamente selecionada.

Para a análise dos dados utilizou-se o pacote estatístico Stata versão 9.2 (Stata Corp., College Station, Estados Unidos). Inicialmente, analisou-se a proporção de adolescentes com prega cutânea elevada de acordo com cada uma das variáveis independentes. Na análise ajustada empregou-se a regressão de Poisson com variância robusta. Essa análise foi realizada de acordo com um modelo hierárquico elaborado previamente (Figura 1). Neste método de análise, as variáveis são controladas para aquelas do mesmo nível ou em níveis superiores 22. Para a manutenção das 
variáveis no modelo, adotou-se como nível de significância um valor $\mathrm{p}<0,20$. Utilizou-se os testes de Wald para heterogeneidade para variáveis dicotômicas ou nominais e de tendência linear para variáveis categóricas ordinais.

O protocolo do estudo foi aprovado pelo Comitê de Ética em Pesquisa da Faculdade de Medicina da Universidade Federal de Pelotas. Foi obtido consentimento informado por escrito dos pais ou responsáveis pelos adolescentes, assim como consentimento verbal por parte do adolescente.

\section{Resultados}

Dos 5.249 adolescentes que iniciaram a coorte em 1993, foram encontrados 4.482 no acompanhamento de 2004-2005. Destes, 30 se recusaram a participar do estudo, totalizando 4.452 adolescentes para a amostra deste estudo (taxa de acompanhamento igual a $87,5 \%$ ). Além das perdas e recusas, houve ainda 24 e 27 perdas para as variáveis PCT e PCS, respectivamente. A variável com o maior número de valores ignorados foi o IMC materno ( $\mathrm{n}=309$, representando $6,9 \%$ do total de adolescentes entrevistados).

A amostra foi composta por aproximadamente $65 \%$ de adolescentes com cor de pele branca, $46 \%$ pertencentes aos níveis econômicos mais baixos (D e E) e 38\% cuja escolaridade foi inferior a quatro anos completos. Cerca de $13 \%$ dos adolescentes possuíam dieta inadequada; $58 \%$ tinham baixa atividade física; e quase $50 \%$ ficavam quatro horas ou mais por dia assistindo à televisão, jogando videogame ou em atividade no computador. A média de idade foi de 11,3 anos (desvio padrão $-\mathrm{DP}=0,3$ ).

Entre os meninos $(\mathrm{N}=2.192)$, o percentual de PCT elevada foi de $20,2 \%$ e de PCS elevada de 17,3\%. A média da PCT e da PCS em milímetros foi, respectivamente, 12,8 (DP = 7,1) e 8,5 (DP = $5,9)$. Entre as meninas $(N=2.260)$, o percentual de prega cutânea elevada foi de $14,2 \%$ para a PCT e de $10,5 \%$ para a PCS. Na amostra total, a média da PCT e da PCS foi, respectivamente, $14,0 \mathrm{~mm}$ (DP = 6,3) e 9,7mm (DP = 5,7).

A Tabela 1 apresenta a análise bruta da associação entre os desfechos e as variáveis independentes para os adolescentes do sexo masculino. A proporção de meninos com PCT e PCS elevadas foi maior entre os adolescentes de cor branca e de maior escolaridade. Percebe-se também que ambas as pregas cutâneas tiveram associação positiva com nível e IMC materno. Dieta inadequada, baixa atividade física e comportamento sedentário não se associaram com nenhuma das pregas cutâneas. Na análise ajustada (Tabela 2), a
Figura 1

Modelo hierárquico de análise para a determinação dos desfechos.

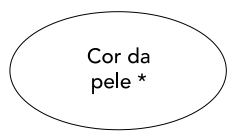

Nível econômico

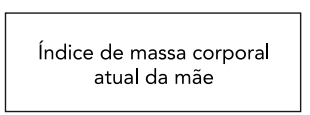

Escolaridade do adolescente
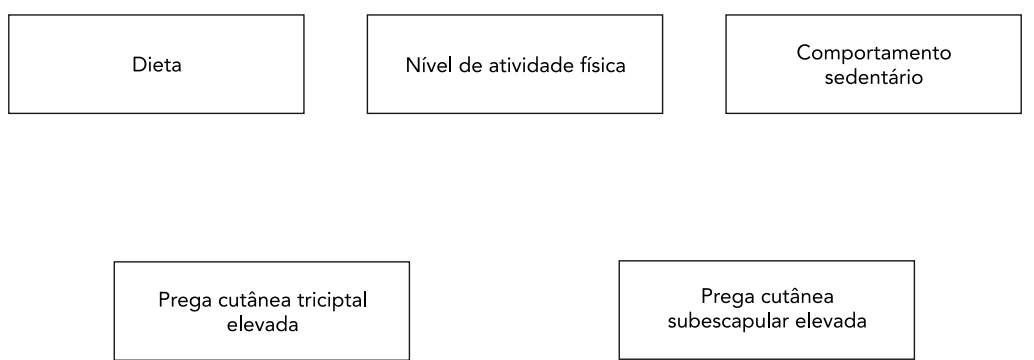

Prega cutânea subescapular elevad

* Variável de ajuste (potencial fator de confusão para todas as demais variáveis incluídas no modelo).

variável cor da pele perdeu a significância. Nível econômico, IMC materno e escolaridade permaneceram fortemente associados com ambas as pregas.

Entre as meninas (Tabela 3), observou-se que o nível econômico e o IMC materno associaram-se com as duas pregas cutâneas. Baixa atividade física foi associada apenas com a PCS elevada enquanto que o comportamento sedentário mostrou-se associado com a PCT. Similarmente ao que ocorreu com os meninos, a PCT e PCS elevadas apresentaram associação com nível econômico e IMC materno, tanto na análise bruta quanto na ajustada (Tabela 4). Já a baixa atividade física esteve associada na análise bruta e ajustada apenas com a PCS. Comportamento sedentário mostrou associação significativa apenas com a PCT elevada na análise bruta, perdendo a significância na análise ajustada.

\section{Discussão}

A distribuição anatômica da gordura é um fator importante na determinação do risco para doen- 
Descrição da amostra e proporção dos adolescentes do sexo masculino com prega cutânea elevada de acordo com as variáveis estudadas.

\begin{tabular}{|c|c|c|c|c|c|}
\hline \multirow[t]{2}{*}{ Variável } & \multirow[t]{2}{*}{$\mathbf{n}$} & \multicolumn{2}{|c|}{$\begin{array}{l}\text { Prega cutânea } \\
\text { triciptal elevada }\end{array}$} & \multicolumn{2}{|c|}{$\begin{array}{c}\text { Prega cutânea } \\
\text { subescapular elevada }\end{array}$} \\
\hline & & $\%$ & Valor $\mathrm{p}$ & $\%$ & Valor $\mathrm{p}$ \\
\hline Cor da pele & & & 0,001 * & & 0,04 * \\
\hline Não-branco & 689 & 16,0 & & 14,7 & \\
\hline Branco & 1.481 & 22,1 & & 18,4 & \\
\hline Nível econômico & & & $<0,001 * \star$ & & $<0,001 * *$ \\
\hline $\mathrm{D} / \mathrm{E}$ (mais pobres) & 996 & 12,8 & & 11,1 & \\
\hline C & 723 & 21,7 & & 20,4 & \\
\hline $\mathrm{A} / \mathrm{B}$ & 452 & 34,2 & & 26,2 & \\
\hline Índice de massa corporal da mãe $\left(\mathrm{kg} / \mathrm{m}^{2}\right) * \star \star$ & & & $<0,001 \star *$ & & $<0,001 * *$ \\
\hline$<25,0$ & 894 & 15,2 & & 12,3 & \\
\hline 25,0 a 29,9 & 671 & 22,3 & & 18,4 & \\
\hline$\geq 30,0$ & 470 & 27,8 & & 25,7 & \\
\hline Escolaridade (anos) & & & $<0,001 *$ & & $<0,001$ * \\
\hline$<4$ & 968 & 13,1 & & 11,8 & \\
\hline$\geq 4$ & 1.216 & 25,6 & & 21,6 & \\
\hline Dieta inadequada & & & 0,5 * & & 0,2 * \\
\hline Não & 1.884 & 20,0 & & 16,9 & \\
\hline Sim & 300 & 21,7 & & 20,0 & \\
\hline Baixa atividade física & & & 0,2 * & & 0,2 * \\
\hline Não & 1.088 & 19,1 & & 16,3 & \\
\hline Sim & 1.029 & 21,4 & & 18,4 & \\
\hline Comportamento sedentário (horas/dia) & & & 0,09 * & & 0,07 * \\
\hline$<4$ & 1.078 & 18,7 & & 15,8 & \\
\hline$\geq 4$ & 1.100 & 21,6 & & 18,7 & \\
\hline Total & 2.192 & 20,2 & & 17,3 & \\
\hline
\end{tabular}

* Teste de Wald para heterogeneidade;

** Teste de Wald para tendência linear;

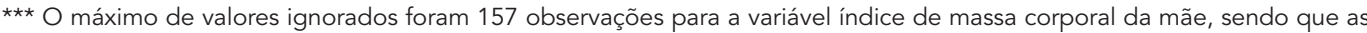
demais variáveis não tiveram mais de 75 valores ignorados.

ças metabólicas e cardiovasculares. O acúmulo de gordura na região visceral merece atenção especial por estar mais fortemente associada com doenças crônico-degenerativas do que a gordura localizada em outras regiões. No presente estudo, a medida da PCS foi utilizada como estimativa de gordura do tronco, enquanto que a PCT foi utilizada para estimar a gordura periférica 23 .

O critério utilizado para definir a presença dos desfechos neste estudo se baseia em padrões descritivos de uma população de adolescentes norte-americanos 16 . Ainda que existam diferenças entre as populações investigadas, acredita-se que isso não tenha afetado o objetivo do estudo, que foi descrever a freqüência de pregas cutâneas tricipital e subescapular elevadas e sua associação com fatores demográficos, sócio-econômi- cos, biológicos e comportamentais na população. Além de o critério adotado ser largamente empregado em outros estudos, a elaboração de padrões normativos para pregas cutâneas em adolescentes não tem uma recomendação clara sobre qual grupo poderia servir como parâmetro apropriado de comparação, o que justifica a utilização de padrões descritivos.

A taxa de perdas de acompanhamento ao longo dos 11 anos do estudo foi de 12,5\%. Considerando a duração do estudo, o percentual de indivíduos encontrados foi relativamente alto. Outro aspecto positivo do estudo é que este é um dos únicos a mensurar pregas cutâneas em uma amostra representativa de adolescentes brasileiros. Além disso, analisaram-se tanto fatores individuais quanto maternos. 
Razão de prevalência (RP) bruta e ajustada de adolescentes do sexo masculino com prega cutânea elevada, de acordo com as variáveis estudadas.

\begin{tabular}{|c|c|c|c|c|c|c|c|c|c|}
\hline \multirow[t]{3}{*}{ Nível } & \multirow[t]{3}{*}{ Variável } & \multicolumn{4}{|c|}{ Prega cutânea triciptal } & \multicolumn{4}{|c|}{ Prega cutânea subescapular } \\
\hline & & \multicolumn{2}{|c|}{ Análise bruta } & \multicolumn{2}{|c|}{ Análise ajustada } & \multicolumn{2}{|c|}{ Análise bruta } & \multicolumn{2}{|c|}{ Análise ajustada } \\
\hline & & RP (IC95\%) & Valor $p$ & RP (IC95\%) & Valor $p$ & RP (IC95\%) & Valor $p$ & RP (IC95\%) & Valor $p$ \\
\hline \multirow[t]{7}{*}{1} & Cor da pele & & 0,001 * & & $0,3^{*}$ & & 0,04 * & & 0,8 * \\
\hline & Não-branco & 1,00 & & 1,00 & & 1,00 & & 1,00 & \\
\hline & Branco & $1,38(1,14-1,68)$ & & $1,12(0,92-1,37)$ & & $1,25(1,01-1,54)$ & & $1,03(0,83-1,27)$ & \\
\hline & Nível econômico & & $<0,001 * \star$ & & $<0,001 \star \star *$ & & $<0,001 * *$ & & $<0,001 * *$ \\
\hline & D/E (mais pobres) & 1,00 & & 1,00 & & 1,00 & & 1,00 & \\
\hline & C & $1,70(1,37-2,10)$ & & $1,70(1,37-2,10)$ & & $1,85(1,47-2,32)$ & & $1,85(1,47-2,32)$ & \\
\hline & $\mathrm{A} / \mathrm{B}$ & $2,68(2,17-3,29)$ & & $2,68(2,17-3,29)$ & & $2,37(1,88-3,00)$ & & $2,37(1,88-3,00)$ & \\
\hline \multirow[t]{8}{*}{2} & Índice de massa & & $<0,001 * \star$ & & $<0,001 * \star$ & & $<0,001 * *$ & & $<0,001 * *$ \\
\hline & corporal da mãe (kg/m²) & & & & & & & & \\
\hline & $<25,0$ & 1,00 & & 1,00 & & 1,00 & & 1,00 & \\
\hline & 25,0 a 29,9 & $1,47(1,19-1,81)$ & & $1,44(1,18-1,77)$ & & $1,50(1,18-1,90)$ & & $1,48(1,17-1,87)$ & \\
\hline & $\geq 30,0$ & $1,83(1,48-2,27)$ & & $2,06(1,67-2,53)$ & & $2,09(1,65-2,64)$ & & $2,35(1,87-2,95)$ & \\
\hline & Escolaridade (anos) & & $<0,001 *$ & & $<0,001 *$ & & $<0,001 *$ & & 0,002 * \\
\hline & $<4$ & 1,00 & & 1,00 & & 1,00 & & 1,00 & \\
\hline & $\geq 4$ & $1,95(1,62-2,36)$ & & $1,55(1,25-1,93)$ & & $1,83(1,49-2,24)$ & & $1,46(1,15-1,85)$ & \\
\hline \multirow[t]{9}{*}{3} & Dieta inadequada & & 0,5 * & & 0,6 * & & 0,2 * & & 0,7 * \\
\hline & Não & 1,00 & & 1,00 & & 1,00 & & 1,00 & \\
\hline & Sim & $1,09(0,86-1,37)$ & & $0,94(0,74-1,20)$ & & $1,19(0,93-1,52)$ & & $1,04(0,81-1,35)$ & \\
\hline & Baixa atividade física & & 0,2 * & & 0,7 * & & 0,2 * & & 0,4 * \\
\hline & Não & 1,00 & & 1,00 & & 1,00 & & 1,00 & \\
\hline & Sim & $1,12(0,95-1,33)$ & & $1,06(0,89-1,26)$ & & $1,12(0,93-1,36)$ & & $1,09(0,90-1,32)$ & \\
\hline & $\begin{array}{l}\text { Comportamento } \\
\text { sedentário (horas/dia) }\end{array}$ & & 0,09 * & & 0,7 * & & 0,07 * & & 0,6 * \\
\hline & $<4$ & 1,00 & & 1,00 & & 1,00 & & 1,00 & \\
\hline & $\geq 4$ & $1,16(0,98-1,37)$ & & $0,97(0,81-1,15)$ & & $1,18(0,98-1,43)$ & & $0,95(0,78-1,15)$ & \\
\hline
\end{tabular}

* Teste de heterogeneidade de Wald;

** Teste de tendência linear de Wald.

A prevalência da PCT elevada foi maior que a PCS em ambos os sexos, sendo que os meninos apresentaram maior prevalência $(20,2 \%$ contra $14,2 \%$ nas meninas). Esse achado sugere que o padrão de acúmulo de gordura em adolescentes dessa idade está mais localizado na região periférica, e que meninos estão acumulando mais gordura do que as meninas. Apesar de o presente estudo ter identificado mais adolescentes com PCT elevada do que PCS, estudos longitudinais apontam que a magnitude do aumento da obesidade central é superior ao da obesidade total durante os primeiros anos da adolescência 24 . O achado desses estudos longitudinais é relevante em termos de saúde pública porque pode representar um aumento da incidência de adolescentes com síndrome metabólica, o que já tem sido observado em países desenvolvidos 25 . A literatura também indica que os fatores de risco para obesidade total e central são similares 24,26. Por exemplo, Garnett et al. 24 verificaram forte associação positiva entre IMC materno e nível de adiposidade do filho(a), consistentemente aos achados do presente estudo.

A variável mais associada tanto com a PCT quanto com a PCS elevada nos meninos foi o nível econômico, com uma forte associação doseresposta $(p<0,001)$. Entre as meninas, mesmo que não tenha havido diferença entre as classes A/B e C, também foi verificada a mesma tendência. Esses achados concordam com um estudo realizado no Canadá 27 , no qual adolescentes de melhor condição sócio-econômica tinham maior nível de adiposidade.

Para os adolescentes do sexo feminino, o mais forte preditor de adiposidade foi o IMC da mãe, também apresentando alto gradiente doseresposta $(p<0,001)$. Meninas cujas mães eram 
Descrição da amostra e proporção de adolescentes do sexo feminino com prega cutânea elevada de acordo com as variáveis estudadas.

\begin{tabular}{|c|c|c|c|c|c|}
\hline \multirow[t]{2}{*}{ Variável } & \multirow[t]{2}{*}{$\mathrm{n}$} & \multicolumn{2}{|c|}{$\begin{array}{l}\text { Prega cutânea } \\
\text { triciptal elevada }\end{array}$} & \multicolumn{2}{|c|}{$\begin{array}{c}\text { Prega cutânea } \\
\text { subescapular elevada }\end{array}$} \\
\hline & & $\%$ & Valor $\mathrm{p}$ & $\%$ & Valor $p$ \\
\hline \multicolumn{2}{|l|}{ Cor da pele } & & 0,06 * & & 0,4 * \\
\hline Não-branco & 778 & 12,4 & & 9,7 & \\
\hline Branco & 1.472 & 15,3 & & 10,9 & \\
\hline \multicolumn{2}{|l|}{ Nível econômico } & & $<0,001 * \star$ & & 0,002 ** \\
\hline D/E (mais pobres) & 1.035 & 10,8 & & 8,1 & \\
\hline C & 791 & 16,9 & & 12,7 & \\
\hline $\mathrm{A} / \mathrm{B}$ & 409 & 17,8 & & 12,4 & \\
\hline \multicolumn{2}{|c|}{ Índice de massa corporal da mãe $\left(\mathrm{kg} / \mathrm{m}^{2}\right)^{\star \star \star}$} & & $<0,001 * \star$ & & $<0,001 * \star$ \\
\hline$<25,0$ & 904 & 9,1 & & 5,7 & \\
\hline 25,0 a 29,9 & 739 & 14,9 & & 11,2 & \\
\hline$\geq 30,0$ & 465 & 22,8 & & 19,2 & \\
\hline \multicolumn{2}{|l|}{ Escolaridade (anos) } & & 0,08 * & & 0,5 * \\
\hline$<4$ & 736 & 12,3 & & 9,9 & \\
\hline$\geq 4$ & 1.522 & 15,1 & & 10,7 & \\
\hline \multicolumn{2}{|l|}{ Dieta inadequada } & & 0,7 * & & 0,7 * \\
\hline Não & 1.962 & 14,3 & & 10,4 & \\
\hline Sim & 296 & 13,6 & & 11,2 & \\
\hline \multicolumn{2}{|l|}{ Baixa atividade física } & & 0,09 * & & 0,02 * \\
\hline Não & 704 & 12,7 & & 8,5 & \\
\hline Sim & 1.466 & 15,4 & & 11,8 & \\
\hline \multicolumn{2}{|c|}{ Comportamento sedentário (horas/dia) } & & 0,03 * & & 0,4 * \\
\hline$<4$ & 1.262 & 12,7 & & 10,0 & \\
\hline$\geq 4$ & 992 & 16,0 & & 11,1 & \\
\hline Total & 2.260 & 14,2 & & 10,5 & \\
\hline
\end{tabular}

* Teste de Wald para heterogeneidade;

** Teste de Wald para tendência linear;

*** O máximo de valores ignorados foram 152 observações para a variável índice de massa corporal da mãe, sendo que as demais variáveis não tiveram mais de 90 valores ignorados.

obesas tiveram 2,6 e 3,4 vezes mais chances de apresentarem PCT e PCS elevadas, respectivamente. Isso reforça a importância das características maternas na composição corporal dos filhos. Esse achado também pode refletir a influência de outros fatores, tais como, hábitos alimentares inadequados e baixo nível de atividade física dos pais e que são adotados pelos filhos, aumentando a chance de sobrepeso/obesidade. Estudos sobre essa relação já foram realizados 28 , entretanto, o presente trabalho não coletou informações sobre hábitos alimentares ou nível de atividade física dos pais.

Em relação à associação entre pregas cutâneas elevadas e cor da pele, encontrou-se um percentual maior das duas pregas cutâneas ele- vadas em adolescentes brancos. Porém, essa associação não foi significativa após ajuste para potenciais fatores de confusão (incluindo o nível econômico). A literatura é controversa quanto à relação entre raça e distribuição da gordura corporal 29,30. Um estudo de revisão demonstrou que indivíduos negros possuem maior acúmulo de gordura intramuscular, no entanto, os indivíduos brancos apresentam maior acúmulo de gordura visceral 31 .

Ao contrário do que a literatura apresenta 32,33, não foi encontrada associação positiva entre dieta inadequada e adiposidade. Isso pode ser explicado pelo delineamento do estudo ser transversal, com possibilidade de haver causalidade reversa. Ou seja, adolescentes com maior 
Razão de prevalência (RP) bruta e ajustada de adolescentes do sexo feminino com prega cutânea elevada, de acordo com as variáveis estudadas.

\begin{tabular}{|c|c|c|c|c|c|c|c|c|c|}
\hline \multirow[t]{3}{*}{ Nível } & \multirow[t]{3}{*}{ Variável } & \multicolumn{4}{|c|}{ Prega cutânea triciptal } & \multicolumn{4}{|c|}{ Prega cutânea subescapular } \\
\hline & & \multicolumn{2}{|c|}{ Análise bruta } & \multicolumn{2}{|c|}{ Análise ajustada } & \multicolumn{2}{|c|}{ Análise bruta } & \multicolumn{2}{|c|}{ Análise ajustada } \\
\hline & & RP (IC95\%) & Valor $p$ & RP (IC95\%) & Valor $p$ & RP (IC95\%) & Valor $p$ & RP (IC95\%) & Valor $p$ \\
\hline \multirow[t]{7}{*}{1} & Cor da pele & & 0,06 * & & 0,4 * & & 0,4 * & & 0,8 * \\
\hline & Não-branco & 1,00 & & 1,00 & & 1,00 & & 1,00 & \\
\hline & Branco & $1,24(0,99-1,55)$ & & $1,11(0,88-1,40)$ & & $1,13(0,87-1,46)$ & & $1,03(0,79-1,35)$ & \\
\hline & Nível econômico & & $<0,001 * \star$ & & $<0,001 \star \star *$ & & $0,002 * \star$ & & 0,002 ** \\
\hline & D/E (mais pobres) & 1,00 & & 1,00 & & 1,00 & & 1,00 & \\
\hline & $\mathrm{C}$ & $1,56(1,23-1,97)$ & & $1,56(1,23-1,97)$ & & $1,56(1,18-2,05)$ & & $1,56(1,18-2,05)$ & \\
\hline & $A / B$ & $1,64(1,25-2,16)$ & & $1,64(1,25-2,16)$ & & $1,52(1,09-2,12)$ & & $1,52(1,09-2,12)$ & \\
\hline \multirow[t]{8}{*}{2} & Índice de massa & & $<0,001 * \star$ & & $<0,001 \star \star *$ & & $<0,001 * *$ & & $<0,001 * *$ \\
\hline & corporal da mãe $\left(\mathrm{kg} / \mathrm{m}^{2}\right)$ & & & & & & & & \\
\hline & $<25,0$ & 1,00 & & 1,00 & & 1,00 & & 1,00 & \\
\hline & 25,0 a 29,9 & $1,63(1,25-2,14)$ & & $1,57(1,20-2,06)$ & & $1,97(1,41-2,76)$ & & $1,89(1,35-2,64)$ & \\
\hline & $\geq 30,0$ & $2,51(1,92-3,27)$ & & $2,55(1,95-3,32)$ & & $3,38(2,44-4,69)$ & & $3,43(2,47-4,74)$ & \\
\hline & Escolaridade (anos) & & 0,08 * & & 0,8 * & & 0,5 * & & 0,6 * \\
\hline & $<4$ & 1,00 & & 1,00 & & 1,00 & & 1,00 & \\
\hline & $\geq 4$ & $1,23(0,98-1,54)$ & & $1,04(0,80-1,35)$ & & $1,09(0,84-1,42)$ & & $0,92(0,69-1,24)$ & \\
\hline \multirow[t]{9}{*}{3} & Dieta inadequada & & 0,7 * & & 0,9 * & & 0,7 * & & 0,6 * \\
\hline & Não & 1,00 & & 1,00 & & 1,00 & & 1,00 & \\
\hline & Sim & $0,95(0,70-1,2)$ & & $0,97(0,71-1,33)$ & & $1,08(0,76-1,53)$ & & $1,11(0,79-1,57)$ & \\
\hline & Baixa atividade física & & 0,09 * & & 0,1 * & & $0,02 * \star$ & & 0,03 * \\
\hline & Não & 1,00 & & 1,00 & & 1,00 & & 1,00 & \\
\hline & Sim & $1,22(0,97-1,53)$ & & $1,20(0,95-1,52)$ & & $1,39(1,05-1,83)$ & & $1,38(1,04-1,85)$ & \\
\hline & $\begin{array}{l}\text { Comportamento } \\
\text { sedentário (horas/dia) }\end{array}$ & & 0,03 * & & 0,4 * & & 0,4 * & & 0,7 * \\
\hline & $<4$ & 1,00 & & 1,00 & & 1,00 & & 1,00 & \\
\hline & $\geq 4$ & $1,26(1,02-1,54)$ & & $1,10(0,89-1,36)$ & & $1,12(0,88-1,43)$ & & $0,95(0,74-1,22)$ & \\
\hline
\end{tabular}

* Teste de heterogeneidade de Wald;

** Teste de tendência linear de Wald.

adiposidade poderiam ter mudado o seu comportamento em relação aos hábitos alimentares e, no momento da entrevista, apresentarem dieta adequada. Outra possível explicação para a ausência dessa associação é o fato de o questionário utilizado para avaliar a dieta não ter sido validado para adolescentes brasileiros. O emprego de instrumentos previamente testados e validados e análises longitudinais, em estudos futuros, possibilitarão interpretar esses resultados com maior segurança.

O baixo nível de atividade física associou-se com adiposidade central (PCS elevada) nas meninas. Porém, não houve associação com a adiposidade periférica (PCT elevada) para nenhum dos sexos. Esse resultado pode ser atribuído ao estágio de maturação sexual dos indivíduos estudados, uma vez que a deposição de gordura central começa a acontecer com maior ênfase após o início da puberdade e ocorre mais precocemente nas meninas do que nos meninos. Deve-se considerar também o possível efeito da causalidade reversa, em que os adolescentes com maior adiposidade poderiam ter adotado a prática regular de atividades físicas. Outro fator que pode ter interferido na ausência dessa associação é o ponto de corte adotado para classificar a atividade física.

A escolaridade do adolescente, por sua vez, esteve independentemente associada com ambas as pregas cutâneas elevadas entre os meninos. Outros estudos também verificaram maior prevalência de obesidade entre os indivíduos com maior escolaridade 3,34. É provável que adolescentes com escolaridade adequada para a idade tenham um nível econômico mais elevado, o que facilita o acesso a alimentos e conseqüentemente aumenta o risco do acúmulo de peso. $\mathrm{O}$ 
comportamento sedentário, por sua vez, teve seu efeito confundido por outros fatores e perdeu a significância estatística na análise ajustada.

Conclui-se, a partir dos resultados obtidos, que a PCT e a PCS elevadas estiveram mais fortemente associadas com características sócio-econômicas e IMC materno do que com características relacionadas ao comportamento atual dos adolescentes. Esse achado ressalta a importância de aspectos do contexto social e familiar sobre a composição corporal de meninos e meninas no início da adolescência. Recomen-

\section{Resumo}

O objetivo deste estudo foi descrever e comparar as pregas cutâneas triciptal (PCT) e subescapular (PCS) de acordo com características demográficas, sócio-econômicas, comportamentais e biológicas em adolescentes de Pelotas, Rio Grande do Sul, Brasil. Realizou-se um estudo transversal aninhado a uma coorte de nascimentos ( $N$ =4.452; média de idade = 11 anos). Os desfechos foram apresentar prega cutânea maior ou igual ao percentil 90 da curva de referência do National Center for Health Statistics. A prevalência de PCT e PCS elevada foi, respectivamente, de 20,2\% e 17,3\% nos meninos e de 14,2\% e 10,5\% nas meninas. O fator mais fortemente associado com adiposidade nos meninos foi o nível econômico $(p<0,001)$ e entre as meninas foi $o$ indice de massa corporal materno $(p<0,001)$. Baixo nível de atividade física $(<300$ minutos/semana) esteve associado com PCS elevadas somente entre as meninas, enquanto que escolaridade se associou à PCT e PCS elevada somente entre os meninos. Dieta, cor da pele e comportamento sedentário não se associaram com nenhum dos desfechos. Concluiu-se que os principais preditores de adiposidade foram características maternas e sócio-econômicas. Recomendam-se investigações utilizando-se outras técnicas de composição corporal para confirmação dos achados deste estudo.

Pregas Cutâneas; Antropometria; Obesidade; Adolescente

\section{Colaboradores}

Todos os autores participaram da redação do artigo. R. P. Duquia e S. C. Dumith realizaram as análises dos dados. A. M. B. Menezes e C. L. Araújo supervisionaram o trabalho de campo. da-se que mais pesquisas sobre a epidemiologia da pregas cutâneas em adolescentes brasileiros sejam realizadas. Assim, será possível comparar os resultados encontrados em diferentes contextos e identificar possíveis heterogeneidades em relação à adiposidade em adolescentes de diferentes regiões do Brasil. Por fim, os dados apresentados neste trabalho podem contribuir para a elaboração de intervenções públicas de saúde visando à prevenção do sobrepeso e da obesidade na adolescência e, conseqüentemente, na vida adulta.

\section{Referências}

1. Clinical guidelines on the identification, evaluation, and treatment of overweight and obesity in adults: the evidence report. National Institutes of Health. Obes Res 1998; 6 Suppl 2:51S-209S.

2. I diretriz brasileira de diagnóstico e tratamento da síndrome metabólica. Arq Bras Cardiol 2005; 84 Suppl 1:2-28.

3. Ministério da Saúde/Ministério do Planejamento Orçamento e Gestão/Instituto Brasileiro de Geografia e Estatística. Pesquisa de orçamentos familiares 2002-2003. Antropometria e análise do estado nutricional de crianças e adolescentes no Brasil. Rio de Janeiro: Instituto Brasileiro de Geografia e Estatística; 2006.

4. Veiga GV, Cunha AS, Sichieri R. Trends in overweight among adolescents living in the poorest and richest regions of Brazil. Am J Public Health 2004; 94:1544-8.

5. Goran MI. Measurement issues related to studies of childhood obesity: assessment of body composition, body fat distribution, physical activity, and food intake. Pediatrics 1998; 101:505-18.

6. Farias ES, Salvador MRD. Antropometria, compo-sição corporal e atividade física de escolares. Rev Bras Cineantropom Desempenho Hum 2005; $7: 21-9$.

7. Fox KR, Peters DM, Sharpe P, Bell M. Assessment of abdominal fat development in young adolescents using magnetic resonance imaging. Int J Obes Relat Metab Disord 2000; 24:1653-9.

8. Wells JC, Victora CG. Indices of whole-body and central adiposity for evaluating the metabolic load of obesity. Int J Obes (Lond) 2005; 29:483-9. 
9. Chiara V, Sichieri R, Martins PD. Sensibilidade e especificidade de classificação de sobrepeso em adolescentes, Rio de Janeiro. Rev Saúde Pública 2003; 37:226-31.

10. Veiga GV, Dias PC, Anjos LA. A comparison of distribution curves of body mass index from Brazil and the United States for assessing overweight and obesity in Brazilian adolescents. Rev Panam Salud Pública 2001; 10:79-85.

11. Goldbacher EM, Matthews KA, Salomon K. Central adiposity is associated with cardiovascular reactivity to stress in adolescents. Health Psychol 2005; 24:375-84.

12. Rodriguez G, Moreno LA, Blay MG, Blay VA, Garagorri JM, Sarria A, et al. Body composition in adolescents: measurements and metabolic aspects. Int J Obes Relat Metab Disord 2004; 28 Suppl 3:S54-8.

13. Brambilla P, Manzoni P, Sironi S, Simone P, Del Maschio A, di Natale B, et al. Peripheral and abdominal adiposity in childhood obesity. Int J Obes Relat Metab Disord 1994; 18:795-800.

14. Victora CG, Araújo CL, Menezes AM, Hallal PC, Vieira MF, Neutzling MB, et al. Methodological aspects of the 1993 Pelotas (Brazil) Birth Cohort Study. Rev Saúde Pública 2006; 40:39-46.

15. Lohman TG. Applicability of body composition techniques and constants for children and youths. Exerc Sport Sci Rev 1986; 14:325-57.

16. World Health Organization. Expert Committee on Physical Status: the use and interpretation of anthropometry. Geneva: World Health Organization; 1995.

17. Associação Brasileira de Empresas de Pesquisa. Critério brasileiro de classificação econômica. São Paulo: Associação Brasileira de Empresas de Pesquisa; 2003.

18. Biddle S, Cavill N, Sallis J. Young and active? Young people and health-enhancing physical activity-evidence and implications. London: Health Education Authority; 1998.

19. Block G. Improving diet methods, improving epidemiologic methods. Ann Epidemiol 1994; 4:257-8.

20. Cameron N. The measurement of human growth. Beckenham: Croom Helm Ltd.; 1984.

21. Ministério da Saúde. Saúde da criança: acompanhamento do crescimento e desenvolvimento infantil. Brasília: Ministério da Saúde; 2002.

22. Victora CG, Huttly SR, Fuchs SC, Olinto MT. The role of conceptual frameworks in epidemiological analysis: a hierarchical approach. Int J Epidemiol 1997; 26:224-7.
23. Willett W. Nutritional epidemiology. 2nd Ed. New York: Oxford University Press; 1998.

24. Garnett SP, Cowell CT, Baur LA, Shrewsbury VA, Chan A, Crawford D, et al. Increasing central adiposity: the Nepean longitudinal study of young people aged 7-8 to 12-13y. Int J Obes (Lond) 2005; 29:1353-60.

25. Duncan GE, Li SM, Zhou XH. Prevalence and trends of a metabolic syndrome phenotype among u.s. adolescents, 1999-2000. Diabetes Care 2004; 27:2438-43.

26. Wardle J, Brodersen NH, Cole TJ, Jarvis MJ, Boniface DR. Development of adiposity in adolescence: five year longitudinal study of an ethnically and socioeconomically diverse sample of young people in Britain. BMJ 2006; 332:1130-5.

27. Janssen I, Boyce WF, Simpson K, Pickett W. Influence of individual- and area-level measures of socioeconomic status on obesity, unhealthy eating, and physical inactivity in Canadian adolescents. Am J Clin Nutr 2006; 83:139-45.

28. Frisancho AR. Prenatal compared with parental origins of adolescent fatness. Am J Clin Nutr 2000; 72:1186-90.

29. He Q, Horlick M, Thornton J, Wang J, Pierson Jr. RN, Heshka S, et al. Sex and race differences in fat distribution among Asian, African-American, and Caucasian prepubertal children. J Clin Endocrinol Metab 2002; 87:2164-70.

30. Lovejoy JC, de la Bretonne JA, Klemperer M, Tulley R. Abdominal fat distribution and metabolic risk factors: effects of race. Metabolism 1996; 45:111924.

31. Torriani M, Grinspoon S. Racial differences in fat distribution: the importance of intermuscular fat. Am J Clin Nutr 2005; 81:731-2.

32. Snethen JA, Broome ME, Cashin SE. Effective weight loss for overweight children: a meta-analysis of intervention studies. J Pediatr Nurs 2006; 21:45-56.

33. Tucker LA, Seljaas GT, Hager RL. Body fat percentage of children varies according to their diet composition. J Am Diet Assoc 1997; 97:981-6.

34. Dutra CL, Araujo CL, Bertoldi AD. Prevalência de sobrepeso em adolescentes: um estudo de base populacional em uma cidade no Sul do Brasil. Cad Saúde Pública 2006; 22:151-62.

Recebido em 21/Nov/2006

Versão final reapresentada em 21/Mar/2007

Aprovado em 28/Mai/2007 